\title{
The Maximum of Randomly Weighted Sums with Long Tails in Insurance and Finance
}

\author{
Yiqing Chen ${ }^{a, *}$ Kai W. $\mathrm{Ng}^{b}$ and Kam C. Yuen ${ }^{b}$ \\ ${ }^{a}$ Department of Mathematical Sciences, the University of Liverpool, Liverpool, L69 7ZL, UK \\ ${ }^{b}$ Department of Statistics and Actuarial Science, The University of Hong Kong, Hong Kong
}

September 28, 2011

\begin{abstract}
In risk theory we often encounter stochastic models containing randomly weighted sums. In these sums, each primary real-valued random variable, interpreted as the net loss during a reference period, is associated with a nonnegative random weight, interpreted as the corresponding stochastic discount factor to the origin. Therefore, a weighted sum of $m$ terms, denoted as $S_{m}^{(w)}$, represents the stochastic present value of aggregate net losses during the first $m$ periods. Suppose that the primary random variables are independent of each other with long-tailed distributions and are independent of the random weights. We show conditions on the random weights under which the tail probability of $\max _{1 \leq m \leq n} S_{m}^{(w)}$ - the maximum of the first $n$ weighted sums - is asymptotically equivalent to that of $S_{n}^{(w)}$ - the last weighted sum.

Keywords: Association; Asymptotics; Long tail; Maximum; Randomly weighted sum; Tail probability; Uniformity

2000 Mathematics Subject Classification: Primary 62E20; Secondary 62H20, $62 \mathrm{P} 05$.
\end{abstract}

\section{Introduction}

Throughout this paper, all limit relationships are for $x \rightarrow \infty$ unless otherwise stated. The relation $a(x) \sim b(x)$ stands for $\lim a(x) / b(x)=1$ while the relations $a(x) \gtrsim b(x)$ and $b(x) \lesssim a(x)$ stand for $\lim \inf a(x) / b(x) \geq 1$. Write $x^{+}=\max \{x, 0\}$ for a real number $x$. For notational convenience we write $\underline{x}_{n}=\left(x_{1}, \ldots, x_{n}\right)^{\prime}$ as a column vector of $n$ dimensions.

A distribution $F$ on $(-\infty, \infty)$ is said to be long tailed, denoted as $F \in \mathcal{L}$, if

$$
\lim _{x \rightarrow \infty} \frac{\bar{F}(x+y)}{\bar{F}(x)}=1
$$

*Correspondence: Yiqing Chen, Department of Mathematical Sciences, the University of Liverpool, Liverpool, L69 7ZL, UK; E-mail: yiqing.chen@liv.ac.uk; Tel.: 44-151-794-4749; Fax: 44-151-794-4754 
for some (or, equivalently, for all ) $y \neq 0$. An important subclass of $\mathcal{L}$ is the subexponential class $\mathcal{S}$. By definition, a distribution $F$ on $[0, \infty)$ is said to be subexponential if the relation

$$
\lim _{x \rightarrow \infty} \frac{\overline{F^{n *}}(x)}{\bar{F}(x)}=n
$$

holds for some (or, equivalently, for all ) $n=2,3, \ldots$, where $F^{n *}$ denotes the $n$-fold convolution of $F$. More generally, a distribution $F$ on $(-\infty, \infty)$ is still said to be subexponential if the distribution $F_{+}(x)=F(x) 1_{(0 \leq x<\infty)}$ is subexponential, where $1_{E}$ denotes the indicator of a set $E$. The monograph Embrechts et al. (1997) gives a nice overview of these and related heavy-tailed distribution classes.

Let $X_{1}, \ldots, X_{n}$ be $n$ mutually independent and real-valued random variables with distributions $F_{1}, \ldots, F_{n}$, respectively. Write $S_{m}=\sum_{k=1}^{m} X_{k}$ for $m=1, \ldots, n$ and

$$
M_{n}=\max _{1 \leq m \leq n} S_{m}
$$

If we interpret each $X_{k}$ as the net loss of an insurance company during period $k$ then $M_{n}$ denotes the maximal net loss during the first $n$ periods. Hence, its tail probability $\operatorname{Pr}\left(M_{n}>x\right), x \geq 0$, is understood as the probability of ruin by time $n$ of the insurance company with an initial surplus $x$. Theorem 2.1 of $\mathrm{Ng}$ et al. (2002) shows that the relation

$$
\operatorname{Pr}\left(M_{n}>x\right) \sim \operatorname{Pr}\left(S_{n}>x\right)
$$

holds under the assumption that $F_{k} \in \mathcal{L}$ for each $k=1, \ldots, n$. This result was earlier contained in Theorem 1 of Sgibnev (1996) for a special case that the distributions $F_{1}, \ldots$, $F_{n}$ are identical belonging to the class $\mathcal{S}$.

A Lévy process $\left\{L_{t}, t \geq 0\right\}$ is a continuous-time stochastic process starting from 0 , with stationary and independent increments and being stochastically continuous. Note that Lévy processes are continuous-time analogues of random walks. Berman (1986) and Willekens (1987) showed that if the Lévy measure of a Lévy process $\left\{L_{t}, t \geq 0\right\}$ is long tailed then, for every $t>0$,

$$
\operatorname{Pr}\left(\sup _{0 \leq s \leq t} L_{s}>x\right) \sim \operatorname{Pr}\left(L_{t}>x\right) .
$$

Relations (1.1) and (1.2) clearly reveal the same flavor. By the way, comparing the tail probabilities of $\sup _{0 \leq s \leq t} L_{s}$ and $L_{t}$ with $t>0$ fixed for a Lévy process $\left\{L_{t}, t \geq 0\right\}$ has been an interesting topic in applied probability. For related recent discussions, we refer the reader to Albin and Sundén (2009) and Braverman (2005), as well as a series of other papers by Braverman.

In this paper, we consider a more practical, but much harder, situation than the two above. As before, let $X_{1}, \ldots, X_{n}$ be $n$ mutually independent and real-valued random variables with distributions $F_{1}, \ldots, F_{n}$, respectively. Let each $X_{k}$ be associated with a nonnegative random weight $W_{k}$ for $k=1, \ldots, n$, where $X_{k}$ can be interpreted as the net loss during period $k$ and $W_{k}$ as the stochastic discount factor from time $k$ to time 0 . Write

$$
S_{m}^{(w)}=\sum_{k=1}^{m} W_{k} X_{k} \quad \text { for } \quad m=1, \ldots, n, \quad M_{n}^{(w)}=\max _{1 \leq m \leq n} S_{m}^{(w)} .
$$


Our goal is to establish a similar relation as (1.1) and (1.2); that is,

$$
\operatorname{Pr}\left(M_{n}^{(w)}>x\right) \sim \operatorname{Pr}\left(S_{n}^{(w)}>x\right) .
$$

The expected result (1.4) not only sheds light on how the tail behavior of the maximum of sums is affected by the long tails of summands but also offers practical value. Usually the calculation of $\operatorname{Pr}\left(M_{n}^{(w)}>x\right)$ is much more laborious than that of $\operatorname{Pr}\left(S_{n}^{(w)}>x\right)$, but relation (1.4) indicates that if we only look at the far right tail of $M_{n}^{(w)}$ then the calculation can be reduced to that of $S_{n}^{(w)}$. Therefore, the study has potential applications in ruin theory and risk management, where randomly weighted sums and their maximum are basic elements of modelling. See Chen (2009) for related discussions. We would like to point out that, under certain stronger conditions on the distributions of the primary random variables (e.g., the distributions $F_{1}, \ldots, F_{n}$ belong to the class $\mathcal{S}$ ), we are able to further establish the relation

$$
\operatorname{Pr}\left(S_{n}^{(w)}>x\right) \sim \sum_{k=1}^{n} \operatorname{Pr}\left(W_{k} X_{k}>x\right) .
$$

However, we shall not spend time on such an easy improvement but focus on comparing the tail probabilities of $M_{n}^{(w)}$ and $S_{n}^{(w)}$ for the long-tailed case.

The rest of this paper consists of three sections. Section 2 presents the main results, Section 3 gives an application in insurance and Section 4 proves the main results after preparing several useful lemmas.

\section{Main Results}

Recall the quantities $S_{m}^{(w)}, m=1, \ldots, n$, and $M_{n}^{(w)}$ introduced in (1.3). As done by Tang and Tsitsiashvili (2003b), we assume that the random weights are bounded away both from 0 and from $\infty$; that is, there are some $0<a \leq b<\infty$ such that

$$
\operatorname{Pr}\left(a \leq W_{k} \leq b\right)=1 \quad \text { for } k=1, \ldots, n .
$$

Theorem 2.1. The relations

$$
\operatorname{Pr}\left(M_{n}^{(w)}>x\right) \sim \operatorname{Pr}\left(S_{n}^{(w)}>x\right) \sim \operatorname{Pr}\left(\sum_{k=1}^{n} W_{k} X_{k}^{+}>x\right)
$$

hold under the following three assumptions:

$P_{1}$ The primary random variables $X_{1}, \ldots, X_{n}$ are mutually independent, with each $X_{k}$ distributed by $F_{k} \in \mathcal{L}$;

$P_{2}$ The random weights $W_{1}, \ldots, W_{n}$ satisfy relation (2.1) for some $0<a \leq b<\infty$;

$P_{3}$ The sequences $\left\{X_{1}, \ldots, X_{n}\right\}$ and $\left\{W_{1}, \ldots, W_{n}\right\}$ are independent. 
Note that in Theorem 2.1 we do not make any assumption on the dependence structure of $\left\{W_{1}, \ldots, W_{n}\right\}$. A closely related result is Theorem 3.1 of Tang and Tsitsiashvili (2003b). Their result is for a special case in which the primary random variables are independent with common subexponential distribution (so that they can show asymptotic formulas as (1.5)).

Next, we aim to weaken the two-sided boundedness condition (2.1) to a one-sided boundedness condition. The cost of doing so is a restriction on the dependence structure of the random weights. Recall that random variables $W_{1}, \ldots, W_{n}$ are said to be (positively) associated if the inequality

$$
\mathrm{E} f_{1}\left(W_{1}, \ldots, W_{n}\right) f_{2}\left(W_{1}, \ldots, W_{n}\right) \geq \operatorname{E} f_{1}\left(W_{1}, \ldots, W_{n}\right) \operatorname{E} f_{2}\left(W_{1}, \ldots, W_{n}\right)
$$

holds for all coordinatewise (not necessarily strictly) increasing functions $f_{1}$ and $f_{2}$ for which the moments involved exist; see Esary et al. (1967) for the introduction of this concept. Trivially, if in the definition above $f_{1}$ is coordinatewise increasing but $f_{2}$ is coordinatewise decreasing, then inequality (2.3) is changed to

$$
\mathrm{E} f_{1}\left(W_{1}, \ldots, W_{n}\right) f_{2}\left(W_{1}, \ldots, W_{n}\right) \leq \mathrm{E} f_{1}\left(W_{1}, \ldots, W_{n}\right) \operatorname{E} f_{2}\left(W_{1}, \ldots, W_{n}\right) .
$$

This dependence structure enjoys some nice properties, as discussed in Esary et al. (1967). In particular, independent random variables are associated. Furthermore, coordinatewise increasing functions of associated random variables are still associated. More precisely, let

$$
\tilde{W}_{l}=g_{l}\left(W_{1}, \ldots, W_{d}\right), \quad l=1, \ldots, n
$$

where each function $g_{l}$ is coordinatewise increasing. If $W_{1}, \ldots, W_{d}$ are associated then so are $\tilde{W}_{1}, \ldots, \tilde{W}_{n}$.

In the second theorem below, motivated by the work of Tang (2006) we assume that the random weights are associated, which is often relevant for financial data. Then we show that the two-sided boundedness condition (2.1) can be weakened to a one-sided boundedness condition as follows: There is some $b>0$ such that

$$
\operatorname{Pr}\left(0 \leq W_{k} \leq b\right)=1 \quad \text { but } \quad \operatorname{Pr}\left(W_{k}=0\right)<1 \quad \text { for each } k=1, \ldots, n \text {. }
$$

Theorem 2.2. The relations in (2.2) hold under the following three assumptions:

$P_{1}$ The primary random variables $X_{1}, \ldots, X_{n}$ are mutually independent, with each $X_{k}$ distributed by $F_{k} \in \mathcal{L}$;

$P_{2}^{\prime}$ The random weights $W_{1}, \ldots, W_{n}$ are associated and satisfy relation (2.5) for some $0<b<\infty$;

$P_{3}$ The sequences $\left\{X_{1}, \ldots, X_{n}\right\}$ and $\left\{W_{1}, \ldots, W_{n}\right\}$ are independent. 


\section{Application to Ruin Theory}

Following the works of Nyrhinen (1999, 2001), Tang and Tsitsiashvili (2003a, 2004) and Goovaerts et al. (2005), we consider a discrete-time insurance model. For $k=1,2, \ldots$, denote by $L_{k}$ the total amount of losses plus other costs and by $P_{k}$ the total amount of premiums, during period $k$. Note that we do not assume that the loss variables or the random premium amounts are identically distributed. Therefore, by suitably choosing their distributions the model can easily account for the effect of inflation or deflation of economy.

Suppose that the insurer invests his/her wealth in a discrete-time financial market consisting of a risk-free bond with a constant force of compound interest $r>0$ per period (so that 1 dollar at time 0 becomes $\mathrm{e}^{r}$ dollars at time 1 ) and a risky stock with a log-return rate $R_{k} \in(-\infty, \infty)$ during period $k$ (so that 1 dollar at time 0 becomes $\mathrm{e}^{R_{k}}$ dollars at time 1 ), $k=1,2, \ldots$. Assume that $\left\{L_{1}, L_{2}, \ldots ; P_{1}, P_{2}, \ldots\right\}$ form a collection of independent random variables and are independent of $\left\{R_{1}, R_{2}, \ldots\right\}$. However, as shown below, the random variables $R_{1}, R_{2}, \ldots$ are not necessarily independent or identically distributed. In the beginning of every period, the insurer invests a proportion $\pi \in[0,1)$ of his/her current wealth in the stock and keeps the rest in the bond. This is the so-called constant investment portfolio in discrete time since the proportion $\pi$ of risky investment does not vary from time to time.

We remark that we have made these assumptions just for simplicity. Actually, our main results enable us to work for more general risk models.

Denote by $U_{m}$ the insurer's wealth at time $m=1,2, \ldots$, with a deterministic initial value $U_{0}=x>0$. Then, recursively,

$$
U_{m}=\left((1-\pi) \mathrm{e}^{r}+\pi \mathrm{e}^{R_{m}}\right) U_{m-1}+\left(P_{m}-L_{m}\right), \quad m=1,2, \ldots
$$

As usual, define the probability of ruin by time $n$ as

$$
\psi(x ; n)=\operatorname{Pr}\left(\min _{1 \leq m \leq n} U_{m}<0 \mid U_{0}=x\right) .
$$

For notational convenience, introduce

$$
X_{k}=L_{k}-P_{k}, \quad Y_{k}=\frac{1}{(1-\pi) \mathrm{e}^{r}+\pi \mathrm{e}^{R_{k}}}, \quad k=1,2, \ldots
$$

The random variable $X_{k}$ is the net loss during period $k$ and the random variable $Y_{k}$ the overall stochastic discount factor from time $k$ to time $k-1$. In the terminology of Tang and Tsitsiashvili (2003a, 2004), we call $X_{1}, X_{2}, \ldots$ insurance risks and call $Y_{1}, Y_{2}, \ldots$ financial risks. The recursive equation in (3.1) can be rewritten as

$$
U_{m}=Y_{m}^{-1} U_{m-1}-X_{m}, \quad m=1,2, \ldots
$$

Iterating (3.3) and multiplying both sides by $\prod_{k=1}^{m} Y_{k}$, we obtain a sequence of stochastic present values,

$$
\widetilde{U}_{0}=x, \quad \widetilde{U}_{m}=x-\sum_{k=1}^{m} X_{k} \prod_{l=1}^{k} Y_{l}, \quad m=1,2, \ldots
$$


It follows that

$$
\psi(x ; n)=\operatorname{Pr}\left(\min _{1 \leq m \leq n} \widetilde{U}_{m}<0 \mid \widetilde{U}_{0}=x\right)=\operatorname{Pr}\left(\max _{1 \leq m \leq n} \sum_{k=1}^{m} X_{k} \prod_{l=1}^{k} Y_{l}>x\right) .
$$

Thus,

$$
\operatorname{Pr}\left(\sum_{k=1}^{n} X_{k} \prod_{l=1}^{k} Y_{l}>x\right) \leq \psi(x ; n) \leq \operatorname{Pr}\left(\sum_{k=1}^{n} X_{k}^{+} \prod_{l=1}^{k} Y_{l}>x\right) .
$$

For the moment we have no idea about how close the two bounds in (3.4) are.

With $W_{k}=\prod_{l=1}^{k} Y_{l}$ for $k=1,2, \ldots$, we see that

$$
\psi(x ; n)=\operatorname{Pr}\left(\max _{1 \leq m \leq n} \sum_{k=1}^{m} W_{k} X_{k}>x\right),
$$

exactly the tail probability of the maximum of finitely many randomly weighted sums. Now we apply Theorem 2.2. Let $Z_{d}=\left(Z_{1}, \ldots, Z_{d}\right)^{\prime}$ be a random vector following a standard multivariate normal distribution of $d$ dimensions. Assume that

$$
\underline{R_{n}}=\underline{\mu_{n}}+A \underline{Z_{d}},
$$

where $\mu_{n}$ is a nonrandom vector of $n$ dimensions and $A$ is a nonrandom $n \times d$ matrix consisting of only nonnegative elements. Therefore, $\underline{R_{n}}$ follows a multivariate normal distribution of $n$ dimensions with mean vector $\mu_{n}$ and covariance matrix $\Sigma=A A^{\prime}$.

Note that $R_{1}, \ldots, R_{n}$, the random components of $R_{n}$, are associated since $Z_{1}, \ldots, Z_{d}$ are independent (hence associated) and $A$ consists of only nonnegative elements. Thus, the random weights $W_{1}, \ldots, W_{n}$ are also associated. The second equality in (3.2) implies that each financial risk $Y_{k}$ is bounded from above by $(1-\pi)^{-1} \mathrm{e}^{-r}$. Thus, the random weights $W_{1}, \ldots, W_{n}$ are also bounded from above. Assume that each loss variable $L_{k}$ follows a longtailed distribution. Then by Lemma 4.3 below, each net loss variable $X_{k}$ follows a long-tailed distribution too. By Theorem 2.2, for each $n=1,2, \ldots$,

$$
\operatorname{Pr}\left(\sum_{k=1}^{n} X_{k} \prod_{l=1}^{k} Y_{l}>x\right) \sim \psi(x ; n) \sim \operatorname{Pr}\left(\sum_{k=1}^{n} X_{k}^{+} \prod_{l=1}^{k} Y_{l}>x\right) .
$$

The relations in (3.5) show that the two bounds in (3.4) should work fine for relatively large values of $x$.

\section{Proofs of the Main Results}

\subsection{Lemmas}

The following first lemma is not only at the core of the present study but also of independent interest in its own right: 
Lemma 4.1. Let $X_{1}, \ldots, X_{n}$ be $n$ independent random variables, with each $X_{k}$ distributed by $F_{k} \in \mathcal{L}$. Then, for arbitrarily fixed $0<a \leq b<\infty$ and $A>0$, the relation

$$
\operatorname{Pr}\left(\sum_{k=1}^{n} w_{k} X_{k}>x-A\right) \sim \operatorname{Pr}\left(\sum_{k=1}^{n} w_{k} X_{k}>x\right)
$$

holds uniformly for all $\underline{w_{n}}=\left(w_{1}, \ldots, w_{n}\right)^{\prime} \in[a, b]^{n}$; that is,

$$
\lim _{x \rightarrow \infty} \sup _{\underline{w_{n}} \in[a, b]^{n}}\left|\frac{\operatorname{Pr}\left(\sum_{k=1}^{n} w_{k} X_{k}>x-A\right)}{\operatorname{Pr}\left(\sum_{k=1}^{n} w_{k} X_{k}>x\right)}-1\right|=0 .
$$

Proof. We follow the proof of Proposition 5.1 of Tang and Tsitsiashvili (2003b) and proceed by induction. For $n=1$,

$$
\begin{aligned}
\limsup _{x \rightarrow \infty} \sup _{w_{1} \in[a, b]}\left|\frac{\operatorname{Pr}\left(w_{1} X_{1}>x-A\right)}{\operatorname{Pr}\left(w_{1} X_{1}>x\right)}-1\right| & \leq \limsup _{x \rightarrow \infty} \sup _{w_{1} \in[a, b]}\left(\frac{\operatorname{Pr}\left(X_{1}>x / w_{1}-A / a\right)}{\operatorname{Pr}\left(X_{1}>x / w_{1}\right)}-1\right) \\
& =\limsup _{x \rightarrow \infty}\left(\frac{\operatorname{Pr}\left(X_{1}>x-A / a\right)}{\operatorname{Pr}\left(X_{1}>x\right)}-1\right) \\
& =0,
\end{aligned}
$$

where the last step is due to $F_{1} \in \mathcal{L}$. This proves relation (4.1) for $n=1$.

Assume that relation (4.1) holds for $n$. We then prove it for $n+1$; that is, the relation

$$
\operatorname{Pr}\left(\sum_{k=1}^{n+1} w_{k} X_{k}>x-A\right) \sim \operatorname{Pr}\left(\sum_{k=1}^{n+1} w_{k} X_{k}>x\right)
$$

holds uniformly for $\underline{w n+1}_{n} \in[a, b]^{n+1}$. When we rewrite (4.2) as

$$
\operatorname{Pr}\left(\sum_{k=1}^{n} \frac{w_{k}}{w_{n+1}} X_{k}+X_{n+1}>\frac{x}{w_{n+1}}-\frac{A}{w_{n+1}}\right) \sim \operatorname{Pr}\left(\sum_{k=1}^{n} \frac{w_{k}}{w_{n+1}} X_{k}+X_{n+1}>\frac{x}{w_{n+1}}\right)
$$

and take into account the two-sided boundedness of the numbers $w_{1}, \ldots, w_{n}$, it is easily understood that one can put $w_{n+1}=1$ in (4.2) without loss of generality. Therefore, it suffices to prove that, uniformly for $\underline{w_{n}} \in[a, b]^{n}$,

$$
\operatorname{Pr}\left(\sum_{k=1}^{n} w_{k} X_{k}+X_{n+1}>x-A\right) \sim \operatorname{Pr}\left(\sum_{k=1}^{n} w_{k} X_{k}+X_{n+1}>x\right) .
$$

Since $A>0$, this is equivalent to proving that, uniformly for $\underline{w_{n}} \in[a, b]^{n}$,

$$
\operatorname{Pr}\left(\sum_{k=1}^{n} w_{k} X_{k}+X_{n+1}>x-A\right) \lesssim \operatorname{Pr}\left(\sum_{k=1}^{n} w_{k} X_{k}+X_{n+1}>x\right) .
$$


By the induction assumption, for every $\varepsilon>0$ there is some $B_{1}>A$ such that

$$
\sup _{x \geq B_{1}} \sup _{\underline{w_{n}} \in[a, b]^{n}} \frac{\operatorname{Pr}\left(\sum_{k=1}^{n} w_{k} X_{k}>x-A\right)}{\operatorname{Pr}\left(\sum_{k=1}^{n} w_{k} X_{k}>x\right)} \leq 1+\varepsilon .
$$

Relying on this $B_{1}>0$, for $x \geq B_{1}$ we derive

$$
\begin{aligned}
\operatorname{Pr}\left(\sum_{k=1}^{n} w_{k} X_{k}+X_{n+1}>x-A\right) & =\left(\int_{-\infty}^{x-B_{1}}+\int_{x-B_{1}}^{\infty}\right) \operatorname{Pr}\left(\sum_{k=1}^{n} w_{k} X_{k}>x-y-A\right) F_{n+1}(\mathrm{~d} y) \\
& =I_{11}(x)+I_{12}(x)
\end{aligned}
$$

and

$$
\begin{aligned}
\operatorname{Pr}\left(\sum_{k=1}^{n} w_{k} X_{k}+X_{n+1}>x\right) & =\left(\int_{-\infty}^{x-B_{1}}+\int_{x-B_{1}}^{\infty}\right) \operatorname{Pr}\left(\sum_{k=1}^{n} w_{k} X_{k}>x-y\right) F_{n+1}(\mathrm{~d} y) \\
& =I_{21}(x)+I_{22}(x) .
\end{aligned}
$$

Clearly,

$$
\frac{\operatorname{Pr}\left(\sum_{k=1}^{n} w_{k} X_{k}+X_{n+1}>x-A\right)}{\operatorname{Pr}\left(\sum_{k=1}^{n} w_{k} X_{k}+X_{n+1}>x\right)} \leq \max \left\{\frac{I_{11}(x)}{I_{21}(x)}, \frac{I_{12}(x)}{I_{22}(x)}\right\} .
$$

First estimate $I_{11}(x) / I_{21}(x)$. By $(4.4)$,

$$
\begin{aligned}
& \sup _{\underline{w_{n}} \in[a, b]^{n}} \frac{I_{11}(x)}{I_{21}(x)} \leq \sup _{y \leq x-B_{1}} \sup _{\underline{w_{n}} \in[a, b]^{n}} \frac{\operatorname{Pr}\left(\sum_{k=1}^{n} w_{k} X_{k}>x-y-A\right)}{\operatorname{Pr}\left(\sum_{k=1}^{n} w_{k} X_{k}>x-y\right)} \\
& =\sup _{x \geq B_{1}} \sup _{\underline{w}_{n} \in[a, b]^{n}} \frac{\operatorname{Pr}\left(\sum_{k=1}^{n} w_{k} X_{k}>x-A\right)}{\operatorname{Pr}\left(\sum_{k=1}^{n} w_{k} X_{k}>x\right)} \\
& \leq 1+\varepsilon .
\end{aligned}
$$

Next estimate $I_{12}(x) / I_{22}(x)$. We have

$$
\begin{aligned}
\frac{I_{12}(x)}{I_{22}(x)}= & \frac{\left(\int_{x-B_{1}}^{x-A}+\int_{x-A}^{\infty}\right) \operatorname{Pr}\left(\sum_{k=1}^{n} w_{k} X_{k}>x-y-A\right) F_{n+1}(\mathrm{~d} y)}{\left(\int_{x-B_{1}}^{x}+\int_{x}^{\infty}\right) \operatorname{Pr}\left(\sum_{k=1}^{n} w_{k} X_{k}>x-y\right) F_{n+1}(\mathrm{~d} y)} \\
\leq & \frac{F_{n+1}\left(x-B_{1}, x-A\right]+\int_{x-A}^{\infty} \operatorname{Pr}\left(\sum_{k=1}^{n} w_{k} X_{k}>x-y-A\right) F_{n+1}(\mathrm{~d} y)}{\int_{x}^{\infty} \operatorname{Pr}\left(\sum_{k=1}^{n} w_{k} X_{k}>x-y\right) F_{n+1}(\mathrm{~d} y)} \\
& =I_{3}(x)+I_{4}(x) .
\end{aligned}
$$


For $I_{3}(x)$, by $F_{n+1} \in \mathcal{L}$ we see that there is a constant $B_{2} \geq B_{1}$ such that

$$
\begin{aligned}
\sup _{x \geq B_{2}} \sup _{\underline{w_{n}} \in[a, b]^{n}} I_{3}(x) & \leq \sup _{x \geq B_{2}} \sup _{w_{n} \in[a, b]^{n}} \frac{F_{n+1}\left(x-B_{1}, x-A\right]}{\operatorname{Pr}\left(\sum_{k=1}^{n} w_{k} X_{k}>0\right) \overline{F_{n+1}}(x)} \\
& \leq \frac{1}{\prod_{k=1}^{n} \overline{F_{k}}(0)} \sup _{x \geq B_{2}} \frac{F_{n+1}\left(x-B_{1}, x-A\right]}{\overline{F_{n+1}}(x)} \\
& \leq \varepsilon
\end{aligned}
$$

and

$$
\sup _{x \geq B_{2}} \frac{\overline{F_{n+1}}(x-A)}{\overline{F_{n+1}}(x)} \leq 1+\varepsilon .
$$

For $I_{4}(x)$, we have

$$
\begin{aligned}
I_{4}(x) & =\frac{\operatorname{Pr}\left(\sum_{k=1}^{n} w_{k} X_{k}>0\right) \overline{F_{n+1}}(x-A)+\int_{-\infty}^{0} \overline{F_{n+1}}(x-y-A) \operatorname{Pr}\left(\sum_{k=1}^{n} w_{k} X_{k} \in \mathrm{d} y\right)}{\operatorname{Pr}\left(\sum_{k=1}^{n} w_{k} X_{k}>0\right) \overline{F_{n+1}}(x)+\int_{-\infty}^{0} \overline{F_{n+1}}(x-y) \operatorname{Pr}\left(\sum_{k=1}^{n} w_{k} X_{k} \in \mathrm{d} y\right)} \\
& \leq \max \left\{\frac{\overline{F_{n+1}}(x-A)}{\overline{F_{n+1}}(x)}, \frac{\int_{-\infty}^{0} \overline{F_{n+1}}(x-y-A) \operatorname{Pr}\left(\sum_{k=1}^{n} w_{k} X_{k} \in \mathrm{d} y\right)}{\int_{-\infty}^{0} \overline{F_{n+1}}(x-y) \operatorname{Pr}\left(\sum_{k=1}^{n} w_{k} X_{k} \in \mathrm{d} y\right)}\right\} \\
& \leq \max \left\{\frac{\overline{F_{n+1}}(x-A)}{\overline{F_{n+1}}(x)}, \sup _{y \leq 0} \frac{\overline{F_{n+1}}(x-y-A)}{\overline{F_{n+1}}(x-y)}\right\} \\
& =\sup _{y \geq x} \frac{\overline{F_{n+1}}(y-A)}{\overline{F_{n+1}}(y)} .
\end{aligned}
$$

Therefore by (4.9),

$$
\sup _{x \geq B_{2}} \sup _{\underline{w_{n}} \in[a, b]^{n}} I_{4}(x) \leq 1+\varepsilon .
$$

Substituting (4.8) and (4.10) into (4.7) yields

$$
\sup _{x \geq B_{2}} \sup _{w_{n} \in[a, b]^{n}} \frac{I_{12}(x)}{I_{22}(x)} \leq 1+2 \varepsilon .
$$

Further substituting (4.6) and (4.11) into (4.5) yields

$$
\sup _{x \geq B_{2}} \sup _{\underline{w_{n}} \in[a, b]^{n}} \frac{\operatorname{Pr}\left(\sum_{k=1}^{n} w_{k} X_{k}+X_{n+1}>x-A\right)}{\operatorname{Pr}\left(\sum_{k=1}^{n} w_{k} X_{k}+X_{n+1}>x\right)} \leq 1+2 \varepsilon .
$$

This last inequality gives the desired result (4.3) since $\varepsilon$ can be arbitrarily small.

An immediate consequence of Lemma 4.1 is the following: 
Lemma 4.2. Under the conditions of Theorem 2.1, the sum $S_{n}^{(w)}=\sum_{k=1}^{n} W_{k} X_{k}$ follows a long-tailed distribution.

Proof. For every fixed $A>0$, by conditioning on $\underline{W_{n}}$ and using Lemma 4.1 we have

$$
\begin{aligned}
\operatorname{Pr}\left(S_{n}^{(w)}>x-A\right) & =\int_{\underline{w_{n}} \in[a, b]^{n}} \ldots \int_{k=1} \operatorname{Pr}\left(\sum_{k}^{n} w_{k}>x-A\right) \operatorname{Pr}\left(\underline{W_{n}} \in \mathrm{d} \underline{w_{n}}\right) \\
& \sim \int_{\underline{w}_{n} \in[a, b]^{n}} \ldots \int_{k=1} \operatorname{Pr}\left(\sum_{k}^{n} w_{k} X_{k}>x\right) \operatorname{Pr}\left(\underline{W_{n}} \in \mathrm{d} \underline{w_{n}}\right) \\
& =\operatorname{Pr}\left(S_{n}^{(w)}>x\right) .
\end{aligned}
$$

Therefore, $S_{n}^{(w)}$ follows a long-tailed distribution.

The last lemma below is a copy of Lemma 4.2 of Tang (2004):

Lemma 4.3. Consider the difference $X=L-P$ in which $L$ and $P$ are two independent random variables with $P$ non-negative. Then $X$ is long tailed if and only if $L$ is long tailed.

\subsection{Proof of Theorem 2.1}

Clearly, $S_{n}^{(w)} \leq M_{n}^{(w)} \leq \sum_{k=1}^{n} W_{k} X_{k}^{+}$. Hence, it suffices to show that

$$
\operatorname{Pr}\left(S_{n}^{(w)}>x\right) \gtrsim \operatorname{Pr}\left(\sum_{k=1}^{n} W_{k} X_{k}^{+}>x\right) .
$$

According to whether or not each $X_{k}$ is nonnegative we partition the whole space $\Omega$ as

$$
\Omega=\bigcup_{K \subset\{1, \ldots, n\}}\left\{X_{k} \geq 0 \text { for all } k \in K \text { and } X_{l}<0 \text { for all } l \notin K\right\}=\bigcup_{K \subset\{1, \ldots, n\}} \Omega_{K} .
$$

Therefore,

$$
\begin{aligned}
\operatorname{Pr}\left(S_{n}^{(w)}>x\right) & =\sum_{\emptyset \neq K \subset\{1, \ldots, n\}} \operatorname{Pr}\left(S_{n}^{(w)}>x, \Omega_{K}\right) \\
& \geq \sum_{\emptyset \neq K \subset\{1, \ldots, n\}} \operatorname{Pr}\left(\sum_{k \in K} W_{k} X_{k}+b \sum_{l \notin K} X_{l}>x, \Omega_{K}\right) .
\end{aligned}
$$

Introduce $\tilde{X}_{k}^{+}=X_{k} \mid\left(X_{k} \geq 0\right)$ and $\tilde{X}_{k}^{-}=-X_{k} \mid\left(X_{k}<0\right)$ for $k=1, \ldots, n$. For each $K \neq \emptyset$, by Lemma 4.2 the sum $\sum_{k \in K} W_{k} \tilde{X}_{k}^{+}$follows a long-tailed distribution. Furthermore, it is independent of $\sum_{l \notin K} W_{l} \tilde{X}_{l}^{-}$. Therefore, by conditioning on $\Omega_{K}$ and using Lemma 4.3 we obtain

$$
\begin{aligned}
\operatorname{Pr}\left(\sum_{k \in K} W_{k} X_{k}+b \sum_{l \notin K} X_{l}>x, \Omega_{K}\right) & \sim \operatorname{Pr}\left(\sum_{k \in K} W_{k} X_{k}>x, \Omega_{K}\right) \\
& =\operatorname{Pr}\left(\sum_{k=1}^{n} W_{k} X_{k}^{+}>x, \Omega_{K}\right) .
\end{aligned}
$$


Substituting (4.14) into (4.13) yields

$$
\operatorname{Pr}\left(S_{n}^{(w)}>x\right) \gtrsim \sum_{\emptyset \neq K \subset\{1, \ldots, n\}} \operatorname{Pr}\left(\sum_{k=1}^{n} W_{k} X_{k}^{+}>x, \Omega_{K}\right)=\operatorname{Pr}\left(\sum_{k=1}^{n} W_{k} X_{k}^{+}>x\right) .
$$

\subsection{Proof of Theorem 2.2}

The same as in the proof of Theorem 2.1, it suffices to show relation (4.12). We formulate the proof into two steps.

Step 1. Assume that the random weights are strictly positive. Our proof in this step is motivated by an idea of Tang (2006) in proving his Theorem 4.1. Let $\delta \in(0, b)$ be arbitrarily fixed but small. By Theorem 2.1,

$$
\operatorname{Pr}\left(S_{n}^{(w)}>x\right) \geq \operatorname{Pr}\left(S_{n}^{(w)}>x, \bigcap_{k=1}^{n}\left(W_{k} \geq \delta\right)\right) \sim \operatorname{Pr}\left(\sum_{k=1}^{n} W_{k} X_{k}^{+}>x, \bigcap_{k=1}^{n}\left(W_{k} \geq \delta\right)\right) .
$$

Since the random weights $W_{1}, \ldots, W_{n}$ are associated and are independent of $X_{1}, \ldots, X_{n}$, recalling (2.4) we have

$$
\begin{aligned}
& \operatorname{Pr}\left(\sum_{k=1}^{n} W_{k} X_{k}^{+}>x\right) \\
= & \operatorname{Pr}\left(\sum_{k=1}^{n} W_{k} X_{k}^{+}>x, \bigcup_{k=1}^{n}\left(W_{k}<\delta\right)\right)+\operatorname{Pr}\left(\sum_{k=1}^{n} W_{k} X_{k}^{+}>x, \bigcap_{k=1}^{n}\left(W_{k} \geq \delta\right)\right) \\
\leq & \operatorname{Pr}\left(\sum_{k=1}^{n} W_{k} X_{k}^{+}>x\right) \operatorname{Pr}\left(\bigcup_{k=1}^{n}\left(W_{k}<\delta\right)\right)+\operatorname{Pr}\left(\sum_{k=1}^{n} W_{k} X_{k}^{+}>x, \bigcap_{k=1}^{n}\left(W_{k} \geq \delta\right)\right) .
\end{aligned}
$$

It follows that

$$
\operatorname{Pr}\left(\sum_{k=1}^{n} W_{k} X_{k}^{+}>x, \bigcap_{k=1}^{n}\left(W_{k} \geq \delta\right)\right) \geq\left(1-\operatorname{Pr}\left(\bigcup_{k=1}^{n}\left(W_{k}<\delta\right)\right)\right) \operatorname{Pr}\left(\sum_{k=1}^{n} W_{k} X_{k}^{+}>x\right) \text {. }
$$

Substituting (4.16) into (4.15) yields

$$
\operatorname{Pr}\left(S_{n}^{(w)}>x\right) \gtrsim\left(1-\operatorname{Pr}\left(\bigcup_{k=1}^{n}\left(W_{k}<\delta\right)\right)\right) \operatorname{Pr}\left(\sum_{k=1}^{n} W_{k} X_{k}^{+}>x\right) .
$$

Since $\delta$ can be arbitrarily small and each $W_{k}$ is strictly positive, this leads to relation (4.12).

Step 2. Now consider the general case where the random weights possibly assign a mass at value zero. Similarly as in the proof of Theorem 2.1, we partition the whole space $\Omega$ according to whether or not each $W_{k}$ is positive, as

$$
\Omega=\bigcup_{K \subset\{1, \ldots, n\}}\left\{W_{k}>0 \text { for all } k \in K \text { and } W_{l}=0 \text { for all } l \notin K\right\}=\bigcup_{K \subset\{1, \ldots, n\}} \Omega_{K}^{*} .
$$


By the result proven in step 1, we obtain

$$
\begin{aligned}
\operatorname{Pr}\left(S_{n}^{(w)}>x\right) & =\operatorname{Pr}\left(S_{n}^{(w)}>x, \bigcup_{K \subset\{1, \ldots, n\}} \Omega_{K}^{*}\right) \\
& =\sum_{\emptyset \neq K \subset\{1, \ldots, n\}} \operatorname{Pr}\left(\sum_{k \in K} W_{k} X_{k}>x, \Omega_{K}^{*}\right) \\
& \gtrsim \sum_{\emptyset \neq K \subset\{1, \ldots, n\}} \operatorname{Pr}\left(\sum_{k \in K} W_{k} X_{k}^{+}>x, \Omega_{K}^{*}\right) \\
& =\sum_{\emptyset \neq K \subset\{1, \ldots, n\}} \operatorname{Pr}\left(\sum_{k=1}^{n} W_{k} X_{k}^{+}>x, \Omega_{K}^{*}\right) \\
& =\operatorname{Pr}\left(\sum_{k=1}^{n} W_{k} X_{k}^{+}>x\right) .
\end{aligned}
$$

Therefore, relation (4.12) still holds.

Acknowledgments. The authors are thankful to an anonymous referee for a careful reading and helpful comments. This research was initiated during a research visit of the first author to the Department of Statistics and Actuarial Science, the University of Hong Kong in summer 2009. She would like to thank the department for its hospitality.

\section{References}

[1] Albin, J. M. P.; Sundén, M. On the asymptotic behaviour of Lévy processes. I. Subexponential and exponential processes. Stochastic Process. Appl. 119 (2009), no. 1, 281-304.

[2] Berman, S. M. The supremum of a process with stationary independent and symmetric increments. Stochastic Process. Appl. 23 (1986), no. 2, 281-290.

[3] Braverman, M. On a class of Lévy processes. Statist. Probab. Lett. 75 (2005), no. 3, 179-189.

[4] Chen, Y. Study on Insurance Risk Models with Subexponential Tails and Dependence Structure. Ph.D. Thesis, University of Hong Kong, 2009.

[5] Embrechts, P.; Klüppelberg, C.; Mikosch, T. Modelling Extremal Events for Insurance and Finance. Springer-Verlag, Berlin, 1997.

[6] Esary, J. D.; Proschan, F.; Walkup, D. W. Association of random variables, with applications. Ann. Math. Statist. 38 (1967), no. 5, 1466-1474.

[7] Goovaerts, M. J.; Kaas, R.; Laeven, R. J. A.; Tang, Q.; Vernic, R. The tail probability of discounted sums of Pareto-like losses in insurance. Scand. Actuar. J. (2005), no. 6, 446-461. 
[8] Ng, K. W.; Tang, Q.; Yang, H. Maxima of sums of heavy-tailed random variables. Astin Bull. 32 (2002), no. 1, 43-55.

[9] Nyrhinen, H. On the ruin probabilities in a general economic environment. Stochastic Process. Appl. 83 (1999), no. 2, 319-330.

[10] Nyrhinen, H. Finite and infinite time ruin probabilities in a stochastic economic environment. Stochastic Process. Appl. 92 (2001), no. 2, 265-285.

[11] Sgibnev, M. S. On the distribution of the maxima of partial sums. Statist. Probab. Lett. 28 (1996), no. 3, 235-238.

[12] Tang, Q. The ruin probability of a discrete time risk model under constant interest rate with heavy tails. Scand. Actuar. J. (2004), no. 3, 229-240.

[13] Tang, Q. Asymptotic ruin probabilities in finite horizon with subexponential losses and associated discount factors. Probab. Engrg. Inform. Sci. 20 (2006), no. 1, 103-113.

[14] Tang, Q.; Tsitsiashvili, G. Precise estimates for the ruin probability in finite horizon in a discrete-time model with heavy-tailed insurance and financial risks. Stochastic Process. Appl. 108 (2003a), no. 2, 299-325.

[15] Tang, Q.; Tsitsiashvili, G. Randomly weighted sums of subexponential random variables with application to ruin theory. Extremes 6 (2003b), no. 3, 171-188.

[16] Tang, Q.; Tsitsiashvili, G. Finite- and infinite-time ruin probabilities in the presence of stochastic returns on investments. Adv. in Appl. Probab. 36 (2004), no. 4, 1278-1299.

[17] Willekens, E. On the supremum of an infinitely divisible process. Stochastic Process. Appl. 26 (1987), no. 1, 173-175. 